\title{
Kinetic Aspects of the Growth of Klebsiella aerogenes with Some Benzenoid Carbon Sources
}

\author{
By D. J. W. GRANT \\ Department of Chemistry, Fourah Bay College, University College of Sierra \\ Leone, Freetown, Sierra Leone*
}

(Accepted for publication 26 August 1966)

SUMMARY

The aerobic growth of Klebsiella aerogenes was studied in chemically defined media consisting of mineral salts (ammonium as $\mathrm{N}$ source) and various benzenoid compounds singly as sole source of carbon+energy. A strain adapted to a defined glucose mineral salts (ammonium as $\mathbf{N}$ source) medium grew readily in concentrations of benzaldehyde, benzoate, $p$-hydroxybenzoate or phenylacetate of the order $10^{-3} \mathrm{M}$ to $10^{-2} \mathrm{M}$. The initial growth rate in benzoate, $p$-hydroxybenzoate or phenylacetate increased and then decreased with increasing concentration of benzenoid compound, according to a relationship which is interpreted by analogy with enzyme inhibition by excess substrate. The kinetics of growth inhibition by the above compounds in glucose salts medium were similar to those in the simplest case of inhibition of an enzyme or heterogeneous catalyst. During repeated subculture in benzoate, $p$-hydroxybenzoate or phenylacetate as sole carbon source, the bacteria showed various adaptive responses which include an immediate decrease in the lag and an increase in the growth rate. As the concentration of benzoate to which the bacteria had become adapted to utilize as sole carbon + energy source was increased, the molar growth yield and the growth rate changed in a related manner and the inhibitory action of benzoate in glucose salts medium decreased.

\section{INTRODUCTION}

Klebsiella aerogenes can utilize a variety of carbon + energy $(C+E)$ sources and in doing so often shows adaptive responses (see Hinshelwood, 1946; Baskett \& Hinshelwood, 1950) which can be interpreted on the basis of kinetic principles (Hinshelwood, 1946; Dean \& Hinshelwood, 1963, 1964). The purpose of the work to be described was to investigate certain kinetic aspects of the growth of $K$. aerogenes in chemically defined media containing a benzenoid compound as sole $C+E$ source; ammonium was the $\mathrm{N}$ source.

\section{METHODS}

Organism. The organism used was Klebsiella aerogenes NCTC 418 (Aerobacter aerogenes) originally obtained from Professor Sir Cyril Hinshelwood's laboratory.

Apparatus. Each culture was contained in a sterile Pyrex $150 \mathrm{~mm}$. boiling tube of $25 \mathrm{~mm}$. diam. sealed with a sterile cotton-wool plug and maintained at $40.0^{\circ}$ in a water bath. Through the cotton-wool plug passed a sterile $1 \mathrm{ml}$. graduated pipette for withdrawing samples of culture and a sterile Pasteur pipette through which filtered

\footnotetext{
* Present address: Department of Pharmacy, University of Nottingham.
} 
air was passed for aeration and agitation. All glassware was thoroughly washed before use.

Media. Each culture developed in $26 \mathrm{ml}$. medium which was prepared by adding to the culture tube $16 \mathrm{ml}$. of a sterile solution of salts and $10 \mathrm{ml}$. of a sterile solution of the $C+E$ source. These solutions were prepared from glass-distilled water and 'AnalaR' grade reagents or recrystallized laboratory reagents. The salts solution consisted of: $\mathrm{Na}_{2} \mathrm{HPO}_{4}, 3.91 \mathrm{~g} . / 1$.; $\mathrm{KH}_{2} \mathrm{PO}_{4}, 1.83 \mathrm{~g} . / 1 . ;\left(\mathrm{NH}_{4}\right)_{2} \mathrm{SO}_{4}, 1.54 \mathrm{~g}$. 11 .; $\mathrm{MgSO}_{4} .7 \mathrm{H}_{2} \mathrm{O}$, $61.7 \mathrm{mg} . / 1$; $\mathrm{FeSO}_{4} .7 \mathrm{H}_{2} \mathrm{O}, 0 \cdot 16 \mathrm{mg}$. $/ 1 . ; \mathrm{pH} 7 \cdot 12$. The solution of the $C+E$ source was either a glucose solution $(50 \mathrm{~g} . / 1$.) or a solution of a benzenoid compound or a solution of a benzenoid compound + glucose ( $50 \mathrm{~g} . / 1$. unless otherwise stated). Solutions of carboxylic acids were neutralized to $\mathrm{pH} 7 \cdot 12$ with sodium hydroxide. In naming the medium after the predominant species, the carboxylate anion $(>99.7$ mole $\%$ ), there is no intention of implying that the anion is the biologically active species. Indeed, the un-ionized molecule of a weak acid is often more active than the corresponding anion in inhibiting the functions of bacteria (Cruess \& Richert, 1929; Goshorn \& Degering, 1938) and of yeasts, fungi, protozoa and other organisms (for a discussion, see Albert, 1965). Since the media were buffered to the same $\mathrm{pH}$ value in all experiments, the concentrations of the active species in a particular medium were constant fractions of the total molar concentration throughout those phases of growth during which measurements were made. Therefore, no matter what the active species were, the conclusions drawn from the experiments on the inhibitory action of benzoate, $p$-hydroxybenzoate and phenylacetate are not invalidated.

Growth was normally terminated by accumulation of toxic waste products, change in $\mathrm{pH}$ value and shortage of oxygen. When it was considered desirable that the lag on subsequent subculture should be as short as possible, exhaustion of glucose was arranged to limit growth. The medium then contained only $0.8 \mathrm{ml}$. of the glucose solution (50 g. 1 .) instead of $10 \mathrm{ml}$. and is referred to as the limited-glucose medium.

Media containing benzenoid substrates were inoculated with a sufficient volume of bacterial suspension to give a defined initial bacterial concentration in the whole medium (see later). The inoculum was either taken directly from a limited-glucose culture or else consisted of a suspension in phosphate buffer of bacteria which had been taken from the same culture and washed twice by centrifugation with phosphate buffer; this buffer (pH 7.12) contained $\mathrm{Na}_{2} \mathrm{HPO}_{4} 6 \cdot 34$ g./1. and $\mathrm{KH}_{2} \mathrm{PO}_{4} 2.96 \mathrm{~g}$./1.

The organism used in these experiments had previously been grown only in meat extract media and in the glucose medium, to which it was fully adapted by repeated subculture. Subculture of bacteria into fresh medium was made daily unless otherwise stated. One subculture corresponded to about 7 generations.

Measurement of bacterial concentration. The bacterial concentration $(M)$ was determined turbidimetrically by means of a Hilger 'Spekker' photoelectric absorptiometer calibrated in terms of the total number of bacteria in a sample taken from a culture in glucose medium just after the end of the logarithmic phase. The bacteria were counted in a Helber counting chamber under a microscope. The $M$ value of a bacterial suspension is thus expressed as the equivalent number of standard-sized living and dead bacteria $/ 10^{-6} \mathrm{ml}$. which would give the same turbidity as the suspension under consideration. The dry weight of bacteria in $1 \mathrm{ml}$. of a suspension of unit bacterial concentration $(M=1.0)$ was $0.48 \mu \mathrm{g}$.

All cultures were initiated with a standard inoculum $(M=3 \cdot 0)$. At various times 
samples were removed from each culture and immediately placed in the $0.5 \mathrm{ml}$. microcell of the absorptiometer for the determination of $M$. From the plot of $\log M$ against the time after inoculation, the lag and the mean generation time of the culture were calculated. Lags less than about $\mathbf{4 0} \mathrm{min}$. were considered to be negligible.

\section{RESULTS}

Benzenoid compounds as sole carbon + energy sources

The original glucose-adapted strain was tested for its ability to grow in media containing various benzenoid compounds as sole carbon + energy $(C+E)$ source. For this, media containing a given $C+E$ source at concentrations from 0.03 to $4 \mathrm{~g}$./l. were inoculated with sufficient unwashed exhausted limited-glucose culture to give the standard inoculum. This was repeated from 2 to 4 times for each benzenoid $C+E$ source. Growth occurred with benzaldehyde, benzoate, $p$-hydroxybenzoate and phenylacetate but not with $o$-hydroxybenzoate, cinnamate, mandelate, benzilate, acetophenone, phenol or resorcinol. Catechol and quinol were also tested but the results were indefinite because these compounds were rapidly oxidized by the air used for aerating the cultures.

There exists the possibility that the compounds which did not support growth under the conditions of the previous experiment might begin to do so after glucose had been used up from a medium containing glucose + benzenoid compound. As a test for this, bacteria adapted to glucose were subcultured in media containing glucose + benzenoid compound and over 8 subcultures, the glucose concentration was decreased stepwise from $1.54 \mathrm{~g}$./1. to zero, while the concentration of the benzenoid compound was simultaneously increased stepwise. The maximum bacterial concentration $\left(M_{s}\right)$ in the stationary phase of each culture was determined. After growth in the presence of cinnamate, mandelate, benzilate, acetophenone, resorcinol or $o$ hydroxybenzoate, the values of $M_{s}$ were never significantly greater than the corresponding values in glucose alone, which confirms that these benzenoid compounds did not support growth. Phenol at $0.077 \mathrm{~g}$. $/ 1$. or greater completely inhibited growth under the conditions described.

\section{Initial growth in benzoate, p-hydroxybenzoate and phenylacetate media}

Initial growth in media containing benzoate, $p$-hydroxybenzoate or phenylacetate as sole $C+E$ source was slower than in the glucose medium and was preceded by a lag. In benzoate medium the lag always exceeded $6 \mathrm{hr}$ but was very variable. Indeed, with benzoate at $1.99 \times 10^{-2} \mathrm{M}$ or greater growth often did not occur, whereas with $1.58 \times 10^{-2} \mathrm{M}$ or less growth always occurred after lags which ranged from $6.4 \mathrm{hr}$ to $187 \mathrm{hr}$ in parallel tests and which appeared to bear little relation to the concentration.

With $p$-hydroxybenzoate and in phenylacetate the initial lag was less variable than with benzoate and tended to increase with concentration. The initial lags with phenylacetate, washed and unwashed inocula are shown in Fig. 1; those with $p$-hydroxybenzoate were between 1 and $4 \mathrm{hr}$ in the range $1.39 \times 10^{-4} \mathrm{M}$ to $5.57 \times 10^{-2} \mathrm{M}$, and $15-30 \mathrm{hr}$ in the range from $8.35 \times 10^{-2} \mathrm{M}$ to $1.11 \times 10^{-1} \mathrm{M}$; no growth occurred in $1.39 \times 10^{-1} \mathrm{M}$ or greater. The data just given for $p$-hydroxybenzoate refer to unwashed inocula. In general, in all media washing the bacteria of the inocula led to longer lags. 
The mean generation time $(T)$ during initial growth in a given concentration of benzoate was variable but less so than the lag. $T$ for a given concentration of $p$ hydroxybenzoate or phenylacetate was somewhat less variable than for benzoate. For each $C+E$ source and at each concentration the average value of $T$ over several experiments is plotted against concentration of $C+E$ source in Fig. 2. The results show essentially the same pattern for different $C+E$ sources. As the concentration was increased from small values, $T$ decreased to a minimum and then increased again as the concentration of $C+E$ source was further increased. This increase in $T$ indicated

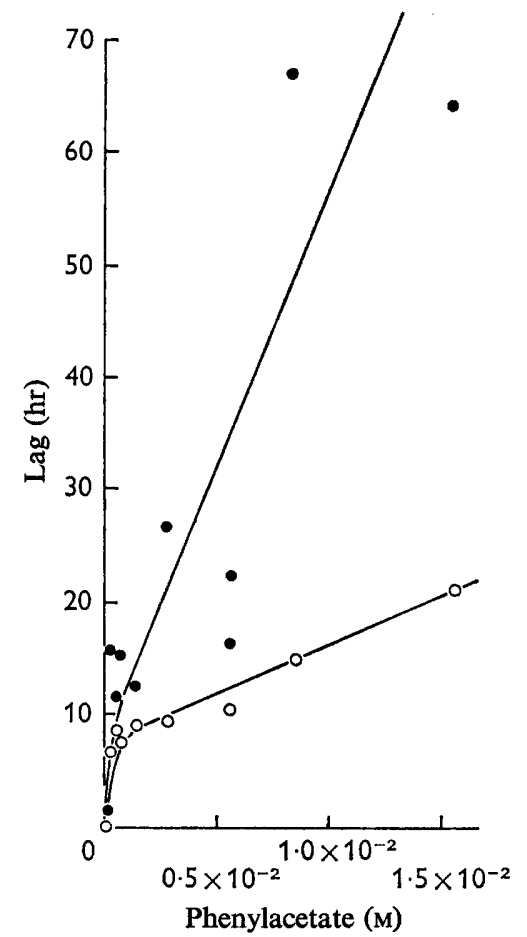

Fig. 1

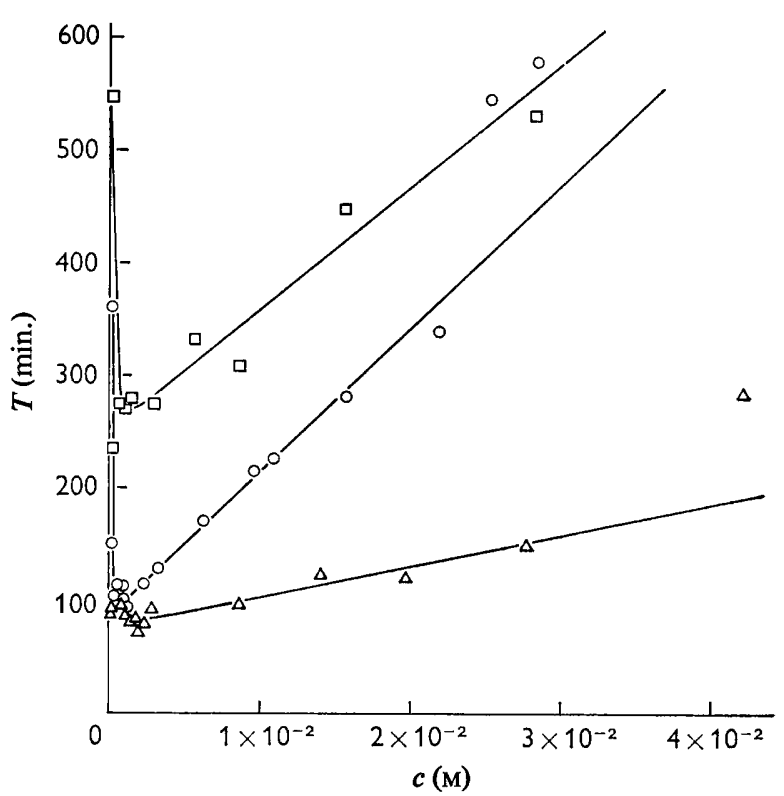

Fig. 2

Fig. 1. Lags preceding initial growth of unwashed (O) and washed (O) Klebsiella aerogenes from the original glucose-adapted strain in a chemically defined medium containing various concentrations of phenylacetate as sole $C+E$ source. (In $2 \cdot 83 \times 10^{-2}$ M-phenylacetate, lag $=$ $135 \mathrm{hr}, \infty$ in separate experiments.)

Fig. 2. Relationships between $T$ of the original glucose-adapted strain of Klebsiella aerogenes and the molar concentration $(c)$ of the following benzenoid compounds, provided as sole $C+E$ source: $O$, benzoate; $\triangle, p$-hydroxybenzoate; $\square$, phenylacetate. (In $5.57 \times 10^{-2} \mathrm{M}$ $p$-hydroxybenzoate, $T=470 \mathrm{~min}$.; in $8.35 \times 10^{-2} \mathrm{M}-p$-hydroxybenzoate, $T=630 \mathrm{~min}$; in $1 \cdot 11 \times 10^{-1} \mathrm{M}-p$-hydroxybenzoate, $T=1280 \mathrm{~min}$.)

that the $C+E$ source was then acting as an inhibitor of growth. In moderate concentrations of each $C+E$ source $T$ was an approximately linear function of the concentration, thus, $T=a+b c$, where $a$ and $b$ are constants for a given $C+E$ source and where $c$ is the molar concentration of the $C+E$ source (Fig. 2). In higher concentrations of benzoate or $p$-hydroxybenzoate (exceeding about 2.5 to $3.0 \times 10^{-2} \mathrm{M}$ ) $T$ increased more and more rapidly with $c$ than is suggested by the linear relationship. 
Serial subculture in benzoate as sole carbon+energy source

The original glucose-adapted strain was subcultivated repeatedly in media containing certain chosen concentrations of benzoate as sole $C+E$ source. After the first subculture in benzoate the lag was never more than $100 \mathrm{~min}$. and during repeated subculture $T$ tended to decrease to an approximately constant minimum value. Whenever the organism was grown in glucose after being subcultured in benzoate, the lag was never more than $60 \mathrm{~min}$. and $T$ remained at its optimum value of 29-33 min.

The original glucose-adapted strain readily became adapted to $9.45 \times 10^{-4} \mathrm{M}$ and $3 \cdot 15 \times 10^{-3} \mathrm{M}$-benzoate but with $9 \cdot 45 \times 10^{-3} \mathrm{M}$ and above it did not grow after 2 or 3 successive subcultures. When trained to $3 \cdot 15 \times 10^{-3} \mathrm{M}$-benzoate it readily became adapted to $9.45 \times 10^{-3} \mathrm{M}$, and when trained to $9.45 \times 10^{-3} \mathrm{M}$ it readily became adapted to $2 \cdot 20 \times 10^{-2} \mathrm{M}$.

When $T$ had become minimal during subcultivation in the chosen concentration of benzoate, adaptation to that concentration was judged to have occurred. Growth curves were then plotted for growth in a number of different concentrations of benzoate, and in the glucose medium as a control. The variation of $T$ with the concentration of benzoate as sole $C+E$ source was of a similar pattern for the various strains trained to benzoate to that for the untrained strain, with the exception that the linear relationship at moderate concentrations of benzoate broke down more and more as the training concentration increased, showing that the adaptive response was rather complex. For example, in the presence of moderate concentrations of benzoate $\left(0-3 \times 10^{-2} \mathrm{M}\right)$, a linear relationship was again found with strains which had been previously trained to benzoate $9.45 \times 10^{-4} \mathrm{M}$ and $3 \cdot 15 \times 10^{-3} \mathrm{M}$. With strains trained to higher concentrations of benzoate, however, the relation between $T$ and $c$ was more complex (Table 1).

\section{Inhibitory effect of certain benzenoid compounds on growth in glucose medium}

The inhibitory actions of $p$-hydroxybenzoate, phenylacetate and benzoate on growth were studied in isolation by plotting growth curves of the organism during incubation in the standard glucose medium to which known amounts of benzenoid compound were added before inoculation.

At low concentrations of $p$-hydroxybenzoate or phenylacetate the lag was negligible (40 min.), but at higher concentrations the lag increased more and more rapidly with increasing concentration (e.g. Fig. 3). Similar variations in the lag with the concentration of other inhibitors have been found (e.g. Hinshelwood, 1946). On the other hand, the lag preceding growth in the glucose medium + benzoate at a range of concentrations $\left(0-5 \cdot 85 \times 10^{-2} \mathrm{M}\right)$ remained negligible.

In glucose medium containing moderate concentrations of benzoate, $p$-hydroxybenzoate or phenylacetate $T$ increased with the concentration of the benzenoid compound according to the general linear relationship, $T=a+b c$, described previously (see Figs. 3, 4; Table 1). The relationship again broke down when the concentration of the inhibitor was high, i.e. when the benzoate exceeded $3 \times 10^{-2} \mathrm{M}$ and the $p$ hydroxybenzoate $8.5 \times 10^{-2} \mathrm{M}$.

The inhibitory action of benzoate on growth in the glucose medium of several strains trained to grow in various concentrations of benzoate as sole $C+E$ source was also investigated. The lag preceding growth in the presence or absence of benzoate was 
negligible and the variation in $T$ was very similar in form to that for the untrained strain (Fig. 4). The gradient of the line, i.e. the constant $b$, for a given strain is a measure of the inhibitory action of benzoate on the growth of that strain in glucose and $b$ tended to decrease as the training concentration of benzoate increased (Table 1). Training to benzoate as sole $C+E$ source therefore tended to decrease the inhibitory action of benzoate on growth in the glucose medium.

Table 1. Values of the constants $a, b$ and $b / a$ in the relationship $T=a+b c$ for certain strains of Klebsiella aerogenes, where $T$ (min.) is the mean generation time of the organism growing in media containing moderate concentrations (c mole/l.) of various single benzenoid compounds

Basal medium: salts +ammonium + carbon source ( $\mathrm{pH} 7 \cdot 12)$. Incubation temperature: $40^{\circ}$.

\begin{tabular}{|c|c|c|c|c|c|c|c|}
\hline \multirow[b]{2}{*}{ Strain } & \multirow[b]{2}{*}{$\begin{array}{l}\text { Benzenoid } \\
\text { compound }\end{array}$} & \multicolumn{3}{|c|}{$\begin{array}{l}\text { Benzenoid compound } \\
\text { as sole carbon + energy } \\
\text { source (nutrient and } \\
\text { inhibitor) }\end{array}$} & \multicolumn{3}{|c|}{$\begin{array}{l}\text { Benzenoid compound } \\
\text { in the glucose medium } \\
\text { (as an inhibitor } \\
\text { only) }\end{array}$} \\
\hline & & $\underset{(\min .)}{a}$ & $\begin{array}{c}b \\
\text { (min. } 1 . \\
\text { mole }^{-1} \text { ) }\end{array}$ & $b / a$ & $\begin{array}{c}a \\
(\min .)\end{array}$ & $\begin{array}{c}b \\
\text { (min. } 1 . \\
\text { mole }^{-1} \text { ) }\end{array}$ & $b / a$ \\
\hline Original glucose-adapted & $\begin{array}{l}p \text {-Hydroxy- } \\
\text { benzoate }\end{array}$ & 75 & 2680 & $35 \cdot 7$ & 29 & 197 & $6 \cdot 8$ \\
\hline Original glucose-adapted & $\begin{array}{l}\text { Phenyl- } \\
\text { acetate }\end{array}$ & 251 & 10800 & $43 \cdot 0$ & 29 & 576 & $19 \cdot 9$ \\
\hline Original glucose-adapted & Benzoate & 88 & 12700 & 144 & 29 & 3100 & 107 \\
\hline $\begin{array}{l}\text { B } 1 \text { trained* to } 9 \cdot 45 \times \\
10^{-4} \mathrm{M} \text {-benzoate }\end{array}$ & Benzoate & 64 & 9670 & 151 & 31 & 3580 & 115 \\
\hline $\begin{array}{l}\text { B2 trained to } 3 \cdot 15 \times \\
10^{-8} \mathrm{M} \text {-benzoate }\end{array}$ & Benzoate & 60 & 6600 & 110 & & 2860 & $95 \cdot 3$ \\
\hline $\begin{array}{l}\text { B3 trained to } 9 \cdot 45 \times \\
10^{-9} \mathrm{M} \text {-benzoate }\end{array}$ & Benzoate & 55 & $\begin{array}{c}\text { Two lin } \\
\text { portion } \\
b=119 \\
1.63 \times \\
b=892 \\
3.75 \times\end{array}$ & $\begin{array}{l}\text { sar } \\
\text { up to } \\
0^{-2} \mathrm{M} \\
\operatorname{up}^{-2} \mathrm{M}\end{array}$ & $30 \cdot 5$ & 2850 & $93 \cdot 5$ \\
\hline $\begin{array}{l}\text { B4 trained to } 2 \cdot 20 \times \\
10^{-2} \mathrm{M} \text {-benzoate }\end{array}$ & Benzoate & 79 & $\begin{array}{l}\text { J-shapec } \\
\text { above } \\
\text { curve o } \\
\text { and cut } \\
\text { curves } \\
\text { and B1 }\end{array}$ & $\begin{array}{l}\text { curve } \\
\text { B3 } \\
\text { ing } \\
\text { f } 2\end{array}$ & $31 \cdot 5$ & 2260 & $71 \cdot 8$ \\
\hline
\end{tabular}

\footnotetext{
* History of strains B1-B 4 of Klebsiella aerogenes trained to utilize benzoate as sole carbon + energy source:

Strain B 1, 30-40 subcultures in $9 \cdot 45 \times 10^{-4} \mathrm{M}$.

Strain B2, 40-50 subcultures in $3.15 \times 10^{-3} \mathrm{M}$.

Strain B 3, 38 subcultures in $3.15 \times 10^{-3} \mathrm{M}$ followed by $70-100$ subcultures in $9.45 \times 10^{-8} \mathrm{M}$.

Strain B4, 38 subcultures in $3 \cdot 15 \times 10^{-3} \mathrm{M}$ followed by 84 subcultures in $9.45 \times 10^{-3} \mathrm{M}$ followed by $40-50$ subcultures in $2.20 \times 10^{-2} \mathrm{M}$.
}

To compare the degre of inhibition (as measured by $b$ ) caused by a given benzenoid compound on the value of $T$ for growth with itself as sole $C+E$ source and for growth with glucose, the value of $T$ at zero inhibition (as measured by $a$ ) must be taken into account. The quantity $b / a$ fulfils this requirement and can be considered to be the 
initial percentage increase in $T$ brought about by an increase in the concentration of benzenoid compound from 0 to $10^{-2} \mathrm{M}$. Table 1 shows that $b / a$ for growth in benzoate, p-hydroxybenzoate or phenylacetate was greater when each compound was sole $C+E$ source than when each was present singly in the glucose medium. Adaptation to low concentrations of benzoate $\left(9.45 \times 10^{-4} \mathrm{M}\right.$ and $\left.3.15 \times 10^{-3} \mathrm{M}\right)$ as sole $C+E$ source did not alter this pattern. Since strains adapted to higher concentrations of benzoate $\left(9.45 \times 10^{-3} \mathrm{M}\right.$ and $\left.2.20 \times 10^{-2} \mathrm{M}\right)$ gave more complex relationships between $T$ and benzoate concentration provided as sole $C+E$ source, $b$ had more than one value over moderate concentrations and so proper comparisons of $b / a$ cannot be made.

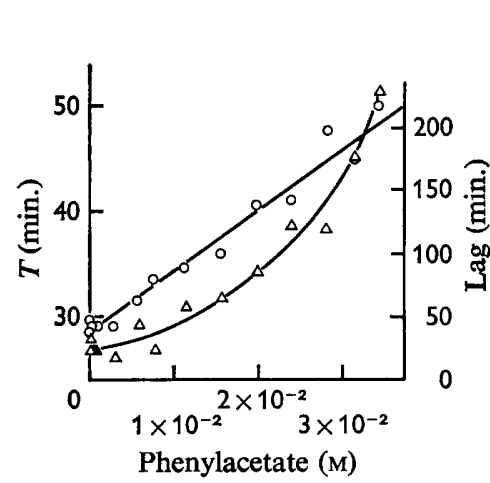

Fig. 3

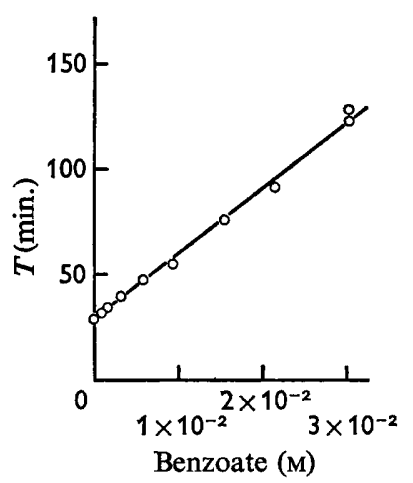

Fig. 4

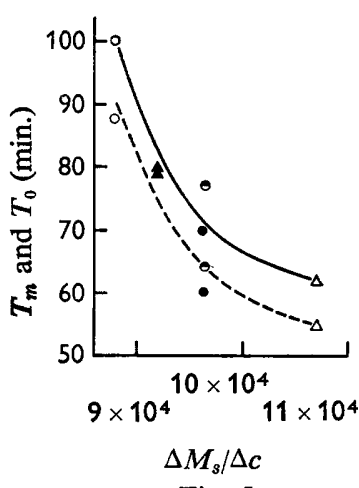

Fig. 5

Fig. 3. Lag $(\triangle)$ and $T(O)$ for the growth of the original glucose-adapted strain of Klebsiella aerogenes in glucose medium containing various concentrations of phenylacetate.

Fig. 4. $T$ for the growth of the original glucose-adapted strain of Klebsiella aerogenes in glucose medium containing various concentrations of benzoate. Lag negligible ( $<40 \mathrm{~min}$.). (In $4.47 \times 10^{-2} \mathrm{M}$-benzoate, lag $=0, T=292 \mathrm{~min}$.; in $5.85 \times 10^{-2} \mathrm{M}$-benzoate, lag $=0, T=$ $1090 \mathrm{~min}$; in $7 \cdot 18 \times 10^{-2} \mathrm{M}$-benzoate, no growth.)

Fig. 5. Relationship between the mean generation time, measured by $T_{0}$ (broken curve) and $T_{m}$ (continuous curve), and the molar growth yield, measured by $\Delta M_{s} / \Delta c$, for the following strains of Klebsiella aerogenes growing in benzoate as sole $C+E$ source. Final training concentration of benzoate (for detailed histories of the strains see Table 1): $O$, untrained; $\Theta, 9.45 \times 10^{-4} \mathrm{M} ; \boldsymbol{\theta}, 3.15 \times 10^{-3} \mathrm{M} ; \triangle, 9.45 \times 10^{-3} \mathrm{M} ; \Delta, 2.20 \times 10^{-2} \mathrm{M}$.

\section{Maximum bacterial concentration $\left(M_{8}\right)$ after exhaustion of a benzenoid carbon +energy source}

When exhaustion of the $C+E$ source limited growth, plots of $M_{s}$ against the initial molar concentration, $c$, of $C+E$ source were linear. At zero substrate concentration the lines intercepted the ordinate at small positive values of $M_{s}$ and there was usually perceptible growth in media to which no $C+E$ source had been added (Bauchop \& Elsden, 1960). This 'impurity' growth accounted for the intercept.

The gradient of the plot of $M_{s}$ against $c\left(\Delta M_{s} / \Delta c\right)$ is termed the molar growth yield (Monod, 1942; Herbert, 1958; Bauchop \& Elsden, 1960), and is a measure of the efficiency with which the particular $C+E$ source is converted to bacterial substance. On general grounds, faster growth might be expected to result in a higher molar growth yield. In fact an inverse correlation between $T$ and $\left(\Delta M_{s} / \Delta c\right)$ was found for strains of Klebsiella aerogenes trained to grow in various concentrations of benzoate as sole $C+E$ source (Fig. 5). The values of $T$ chosen for this plot must be those at which the concentration of $C+E$ source is neither so great as to slow down growth by 
inhibition, nor so small as to limit the growth rate by starvation. The following two attempts were made to fulfil these requirements. The values of $T$ corresponding to the upper (continuous) curve of Fig. 5 are the minimum values $\left(T_{m}\right)$ interpolated from the plots of $T$ against $c$ and those corresponding to the lower (broken) curve are the hypothetical values $\left(T_{0}\right)$ extrapolated to zero concentration from the inhibitory portions of the plots of $T$ against $c . T_{0}$ is thus equal to the constant $a$ in Table 1 . The interpolated quantity $T_{m}$ can be determined with greater accuracy than the extrapolated quantity $T_{0}$ but $T_{0}$ is theoretically preferable because the inhibition is then strictly zero.

On account of the complexities of adaptation, the inverse correlation between $T$ and the molar growth yield for strains in various stages of training to benzoate, when it is acting both as an inhibitor and as a nutrient, could hardly be as simple as the relationship found when a given strain is grown under closely similar conditions within a restricted range (Pirt, 1965). Indeed, Table 2 shows that there was no correlation between $T_{m}$ or $T_{0}$ and the molar growth yield for the original glucose-adapted strain growing with various benzenoid compounds as sole $C+E$ source, no doubt because the conditions of growth were so widely different. The molar growth yield for glucose was, however, higher than that for the benzenoid $C+E$ sources.

Table 2. Molar growth yield (as measured by $\Delta M_{s} \mid \Delta c$ ) and $T$ of the original glucoseadapted strain of Klebsiella aerogenes growing with single benzenoid carbon + energy $(C+E)$ sources

Basal medium: salts + ammonium + carbon source ( $\mathrm{pH} 7 \cdot 12)$. Incubation temperature: $40^{\circ}$.

$\begin{array}{lccc}\text { Carbon source } & \Delta M_{s} / \Delta c & T_{m} & T_{0} \\ \text { Glucose } & 14 \cdot 2 \times 10^{4} & 30 & 30 \\ p \text {-Hydroxybenzoate } & 6.83 \times 10^{4} & 80 & 75 \\ \text { Phenylacetate } & 10.6 \times 10^{4} & 286 & 251 \\ \text { Benzoate } & 8.80 \times 10^{4} & 100 & 88 \\ \text { maximum bacterial concentration in the stationary phase. } & \\ \text { molar concentration of } C+E \text { source. } & \\ \text { minimum value of the mean generation time. } \\ \text { value of the mean generation time extrapolated to zero concentration of } C+E \text { source. }\end{array}$

\section{Serial subculture in p-hydroxybenzoate and phenylacetate}

A glucose-adapted strain which had not been in contact with any other chemically defined medium was repeatedly subcultivated in a medium containing a suitable fixed concentration of the appropriate benzenoid compound as sole $C+E$ source, and the lag and $T$ during growth in both the benzenoid and glucose media were determined at various stages of training. During repeated subculture in the $p$-hydroxybenzoate medium (Table 3 ) the decrease in $T$ to about $45 \mathrm{~min}$. and the decrease in the lag after the first subculture indicated that adaptation to the $C+E$ source was taking place. Table 3 also shows that adaptation to $p$-hydroxybenzoate disturbed growth in glucose medium. Thus partial adaptation increased the value of $T$ in glucose, whereas on prolonged adaptation $T$ was normal but the lag increased.

During subcultivation in medium containing phenylacetate as sole $C+E$ source (Table 4) the sudden decrease in the lag from $700 \mathrm{~min}$. to zero indicated that appreciable adaptation had taken place during the previous subculture. During repeated 
subculture in phenylacetate medium $T$ increased at first and then decreased later. When subculture was made daily (series A) the final value of $T$ was about one half the initial value, but with subculture every second day $T$ did not decrease below the value obtained at the first subculture (series B).

Table 4 shows that during subcultivation in phenylacetate medium the small or zero lag on return to glucose medium was maintained but the value of $T$ in glucose medium was increased, although in series $\mathrm{A}$ it showed some tendency to decrease as the value of $T$ in phenylacetate medium decreased. Subculture in medium containing phenylacetate as sole $C+E$ source was therefore incompatible with optimum growth in glucose medium.

Table 3. Growth of Klebsiella aerogenes during serial subculture with $2 \cdot 78 \times 10^{-2} M$ p-hydroxybenzoate as sole carbon +energy source

Basal medium: salts +ammonium $+p$-hydroxybenzoic acid (pH 7·12). Incubation temperature: $40^{\circ}$. No. of previous

daily Growth in $p$-hydroxybenzoate Growth on return to glucose subcultures in p-hydroxybenzoate before test

$\overbrace{\begin{array}{c}\mathrm{Lag} \\ \text { (min.) }\end{array}}^{\begin{array}{c}T \\ (\mathrm{~min} .)\end{array}}$

$\overbrace{\substack{\text { Lag } \\ \text { (min.) }}}^{T} \begin{array}{c}T \\ \text { (min.) }\end{array})$

$\begin{array}{rrrrc}0 & 100 & 82 & 2 & 33 \\ 1 & 51 & 50 & 60 & 36 \\ 3 & 35 & 47 & 87 & 58 \\ 10 & 0 & 76 & 0 & 78 / 40^{*} \\ 15 & 18 & 54 & 0 & 76 / 37^{*} \\ 28 & 57 & 44 & 89 & 56 / 35^{*} \\ 31 & 24 & 44 & 193 & 33 \\ 43 & 45 & 46 & 175 & 33\end{array}$

* Sudden decrease in $T$ during growth.

Table 4. Growth of Klebsiella aerogenes during serial subculture in $2.83 \times 10^{-2} M$ phenylacetate as sole carbon +energy source

Basal medium: salts + ammonium + phenylacetic acid (pH 7·12). Incubation temperature: $40^{\circ}$.

\begin{tabular}{|c|c|c|c|c|}
\hline \multirow{2}{*}{$\begin{array}{l}\text { No. of previous } \\
\text { subcultures in } \\
\text { phenylacetate } \\
\text { before test }\end{array}$} & \multicolumn{2}{|c|}{ Growth in phenylacetate } & \multicolumn{2}{|c|}{ Growth on return to glucos } \\
\hline & Lag (min.) & $T$ (min.) & Lag (min.) & $T(\min$ \\
\hline \multicolumn{5}{|c|}{ Series A. Subcultured each day } \\
\hline 0 & 645 & 166 & 2 & 33 \\
\hline 3 & 0 & 208 & 0 & 51 \\
\hline 8 & 0 & 104 & 0 & 97 \\
\hline 12 & 0 & 132 & 0 & 86 \\
\hline 22 & 0 & $135 / 88^{*}$ & 0 & 45 \\
\hline 25 & 0 & 83 & 30 & 38 \\
\hline \multicolumn{5}{|c|}{ Series B. Subcultured every 2 days } \\
\hline 0 & 708 & 151 & 60 & 32 \\
\hline 1 & 0 & 150 & - & - \\
\hline 7 & 0 & 159 & 0 & 63 \\
\hline 16 & 0 & 213 & - & - \\
\hline 18 & 0 & 235 & 44 & 47 \\
\hline 35 & 0 & 150 & 0 & 52 \\
\hline 40 & 0 & 159 & 0 & 62 \\
\hline
\end{tabular}

* Sudden decrease in $T$ during growth. 


\section{DISCUSSION}

When Klebsiella aerogenes NCTC418 grew in medium containing glucose and benzoate, $p$-hydroxybenzoate or phenylacetate or when the organism utilized one of these aromatic compounds as sole $C+E$ source, then the following equation was found to relate the mean generation time $(T)$ to the molar concentration $(c)$ of the aromatic compound:

$$
T=a+b c,
$$

where $a$ is the value of $T$ at zero concentration of inhibitor, $b$ is a constant independent of $c$, and $c$ can have values which lie within the limit specified. The specific growth rate, $v(=\ln 2 / T)$, therefore obeys the following relationship:

$$
v=d /(1+e c),
$$

where $d(=\ln 2 / a)$ is the value of $v$ at zero concentration of inhibitor and $e(=b / a)$ is a constant independent of $c$. Equation (2) is also the expression for the rate of reactions catalysed by enzymes or by heterogeneous catalysts in the presence of an inhibitor whose concentration or partial pressure in the bulk phase is represented by $c$, where $d$ and $e$ are constants with the same interpretation as above (see Hinshelwood, 1940; Dixon \& Webb, 1958; Ashmore, 1963). Some apparently more complex expressions for enzyme inhibition reduce to equation (2) or can be made to do so by making suitable approximations.

For the initial growth in benzoate, $p$-hydroxybenzoate or phenylacetate the plot of $T(=\ln 2 / v)$ against the molar concentration of the $C+E$ source (Fig. 2) closely resembles the plot of the reciprocal of the rate of an enzyme-catalysed reaction against the molar concentration of the substrate which simultaneously acts as an inhibitor (see the graphical representation of the data of Murray (1930) for the hydrolysis of ethyl butyrate by sheep liver carboxylesterase, and of the data of Dixon, Massey and Webb for the oxidation of leucine by methylene blue with snake venom L-amino acid oxidase in Dixon \& Webb (1958)). Figure 2 and the plots by Dixon \& Webb (1958) are similar both in form and in the order of magnitude of the molar concentration of the substrate corresponding to the minima.

The above similarities could arise from analogies between an enzyme-catalysed reaction proceeding in vitro and an actively growing bacterial culture. Both processes take place in solution containing various chemical species, among which are either the substrate(s) of the enzyme or the analogous nutrients for the bacteria. An enzyme reaction can be written as follows:

$$
\text { reactants + enzyme } \rightarrow \text { enzyme + products, }
$$

whereas bacterial growth can be represented thus:

nutrients + bacterium $\rightarrow$ new bacterium + (metabolic products + more new bacteria).

Both are slowed down by inhibitors which can act on one or more elementary physical or chemical processes or steps within the enzyme reaction or within the growing cell. The kinetics of inhibition are therefore essentially the same in these two cases and the inhibitor can be a nutrient for the bacteria, a substrate for the enzyme or a foreign substance. 
The simplicity of the observed kinetics of inhibition of bacterial growth summarized by equations ( 1 ) or (2) is readily explicable if benzoate, $p$-hydroxybenzoate and phenylacetate are each acting as an inhibitor of a cellular process or reaction whose rate determines the rate of multiplication of the bacteria. The inhibited process might be not only an enzyme-catalysed metabolic reaction but also membrane transport catalysed by a permease, and this need not necessarily be the same for different benzenoid inhibitors.

As the concentration of the inhibitory compound is increased beyond a certain value, the lag rapidly increases and the mean generation time usually increases more steeply with concentration than is predicted by the linear relationship obeyed at lower concentrations. These changes are observed no matter whether the $C+E$ source is glucose or solely the benzenoid compound itself and suggest that other processes or reactions now begin to be inhibited also and determine the rate of growth.

The increase of lag that results from washing the bacteria of the inoculum may be due to the removal of diffusible metabolites needed for the biochemical reactions involved in cell division (Hinshelwood, 1946; Dagley, Dawes \& Morrison, 1950).

Since the lag preceding growth of the original glucose-adapted strain in glucose media containing a wide range of concentrations of benzoate $\left(0-5.85 \times 10^{-2} \mathrm{M}\right)$ remained negligible, the appreciable lag preceding initial growth in even the lowest concentrations of benzoate as sole $C+E$ source cannot be a consequence of the inhibitory nature of benzoate, and would appear to be a result of the temporary inability of the cells to metabolize the compound.

Repeated subculture in either benzoate, $p$-hydroxybenzoate or phenylacetate as sole $C+E$ source calls forth the usual pattern of adaptive responses which are observed for aliphatic carbon sources (e.g. Hinshelwood, 1946; Baskett \& Hinshelwood, 1950). Thus, the lag decreases to a small or negligible value after the first subculture and the mean generation time eventually decreases to a steady minimum value during repeated subculture in a constant concentration of benzoate or $p$-hydroxybenzoate. Adaptation to benzoate as sole $C+E$ source is at every stage fully compatible with optimum growth in glucose, whereas adaptation to $p$-hydroxybenzoate disturbs growth in glucose. During repeated subcultivation in phenylacetate growth in glucose was impaired.

Comparison of the values of $b / a$ in Table 1 shows that benzoate, $p$-hydroxybenzoate and phenylacetate were stronger inhibitors when they were supporting growth than when glucose was. Furthermore, adaptation to moderate concentrations of benzoate as sole $C+E$ source did not change this pattern, and therefore the relatively greater sensitivity of benzoate metabolism cannot be ascribed to its incomplete development but was probably a consequence of differences inherent in the metabolism of glucose and benzoate.

The molar growth yield for glucose was greater than that for the benzenoid compounds. This cannot be entirely ascribed to the fact that the strain of Klebsiella aerogenes used was already fully adapted to utilize glucose as sole $C+E$ source, because, after the organism had become adapted to benzoate as sole $C+E$ source, the molar growth yield increased by not more than $21 \%$ and was still much smaller than it was for growth in glucose. The metabolism of glucose must therefore be intrinsically more efficient than that for the benzenoid compounds.

As the constant concentration of benzoate which the organism had been trained to 
utilize was increased, the following adaptive responses occurred: (1) the inhibitory action of benzoate on growth in the glucose medium tended to decrease (Table 1), showing that the glucose metabolism of the organism had become less sensitive to inhibition by benzoate; (2) the molar growth yield in benzoate increased somewhat and the uninhibited value of the mean generation time decreased provided that the concentration used for training did not exceed about $10^{-2} \mathrm{M}$ (Fig. 5). Although training to $2 \cdot 20 \times 10^{-2} \mathrm{M}$-benzoate did not affect growth in glucose and further decreased the inhibitory action of benzoate in glucose media, it damaged the metabolism of benzoate as sole $C+E$ source. This is shown by the decrease in the molar growth yield and the increase in the uninhibited value of the mean generation time (Fig. 5) and by the upward sweep of the J-shaped curve connecting the mean generation time and the concentration of benzoate provided as sole $C+E$ source (Table 1).

The author wishes to thank Professor S. R. Landor and colleagues of the Department of Chemistry, University College of Sierra Leone, for helpful discussions and Dr A. C. R. Dean and Professor M. W. Partridge for valuable advice on the preparation of the text.

\section{REFERENCES}

Albert, A. (1965). Selective Toxicity, 3rd edn. London: Methuen.

Ashmore, P. G. (1963). Catalysis and Inhibition of Chemical Reactions. London: Butterworth.

BASKetT, A. C. \& Hinshelwood, C. N. (1950). The utilization of carbon sources by Bact. lactis aerogenes. I. General survey. Proc. R. Soc. B 136, 520.

BAUCHOP, T. \& ElsDEN, S. R. (1960). The growth of micro-organisms in relation to their energy supply. J. gen. Microbiol. 23, 457.

Cruess, W. V. \& RicherT, P. M. (1929). Effects of hydrogen ion concentration on the toxicity of sodium benzoate to micro-organisms. J. Bact. 17, 363.

DAGLeY, S., DAwes, E. A. \& MORRISON, G. A. (1950). Factors influencing the early phases of growth of Aerobacter aerogenes. J. gen. Microbiol. 4, 437.

DeAN, A. C. R. \& Hinshelwood, C. N. (1963). Integration of cell reactions. Nature, Lond. $199,7$.

Dean, A. C. R. \& Hinshelwood, C. N. (1964). Some basic aspects of cell regulation. Nature, Lond. 201, 232.

Dixon, M. \& WebB, E. C. (1958). Enzymes. London: Longmans Green.

Goshorn, R. H. \& Degering, E. F. (1938). Preparation of alpha-phenylalkanoic acids and a study of their bactericidal and physical properties. J. Am. pharm. Ass. 27, 865 .

Herbert, D. (1958). Some principles of continuous culture. In Recent Progress in Microbiology. Ed. by G. Tunevall. Symp. 7th int. Congr. Microbiol., p. 381.

Hinshelwood, C. N. (1940). The Kinetics of Chemical Change. Oxford: Clarendon Press.

Hinshelwood, C. N. (1946). The Chemical Kinetics of the Bacterial Cell. Oxford: Clarendon Press. Monod, J. (1942). Recherches sur la Croissance des Cultures Bactériennes. Paris: Herman et Cie.

Murray, D. R. P. (1930). The inhibition of esterases by excess substrate. Biochem. J. 24, 1890.

PIRT, S. R. (1965). The maintenance energy of bacteria in growing cultures. Proc. R. Soc. B 163, 224. 